\title{
Radiobiological evaluation of dose calculation algorithms in RapidArc planning of esophageal cancer treatment plans
}

\author{
Suresh Rana ${ }^{1}$, Kevin Rogers ${ }^{2}$ \\ 1. Department of Medical Physics, ProCure Proton Therapy Center, Oklahoma City, OK, USA. 2. Department of Radiation \\ Oncology, Arizona Center for Cancer Care, Peoria, AZ, USA
}

Correspondence: Suresh Rana. Address: 5901 West Memorial Road, Oklahoma City, OK, 73142, USA.

E-mail: suresh.rana@gmail.com

Received: April 21, 2013

Accepted: May 16, 2013

Online Published: May 20, 2013

DOI : $10.5430 /$ jst.v3n3p44

URL: http://dx.doi.org/10.5430/jst.v3n3p44

\section{Abstract}

Purpose: The purpose of current study is to investigate the impact of tissue heterogeneity corrections in Acuros XB algorithm (AXB) and Anisotropic Analytical Algorithm (AAA) on RapidArc esophageal cancer treatment plans using equivalent uniform dose (EUD) calculations and tumor control probability (TCP).

Methods: Ten esophageal cancer cases were selected for the current study. All cases were planned using RapidArc technique in Eclipse treatment planning system (10.0.28). The treatment plans were inversely optimized using progressive resolution optimizer and each optimized plan was calculated using AAA with tissue heterogeneity correction. The AAA plans were then normalized such that prescription dose covered $95 \%$ of the planning target volume (PTV). The AXB plans were generated by recalculating the normalized AAA plans using AXB with tissue heterogeneity correction for identical monitor units and beam parameters as in the corresponding AAA plans. The EUD and TCP calculations were then performed on the AAA and AXB plans.

Results: For PTV, the EUD values in AXB plans were always lower than in AAA plans with an average difference of $1.3 \%$. Similarly, AXB calculations produced smaller magnitude of EUD for the organs at risk (OARs) compared to AAA calculations. On average, in AXB plans, the lung EUD was lower by 3.5\%, the liver EUD was lower by 2.3\%, the heart EUD was lower by $2.8 \%$, and the spinal cord EUD was lower by $1.8 \%$. The TCP of AXB plans was lower by average difference of $7.1 \%$ compared to that of AAA plans.

Conclusion: In comparison to AAA calculations, the preliminary results presented in the current study showed that AXB calculations produced lower EUD and TCP for the PTV as well as lower EUD for OARs in the esophageal cancer treatment plans created by RapidArc planning technique.

\section{Key words}

Acuros XB, Anisotropic analytical algorithm, Equivalent uniform dose, Tumor control probability, Esophageal cancer, RapidArc planning

\section{I ntroduction}

Currently, there are an increasing number of radiation oncology departments that are implementing and using volumetric modulated arc therapy (VMAT) technique for external beam radiation therapy. RapidArc (Varian Medical Systems, Palo 
Alto, CA) is an example of volumetric modulated arc therapy (VMAT) technique, which delivers modulated radiation beams with simultaneous adjustment of multi-leaf collimator (MLC) field aperture, dose rate and gantry rotation speed ${ }^{[1]}$. The main goal of RapidArc treatment is to deliver a conformal radiation dose distribution to the target while minimizing the dose to the organs at risk (OARs) ${ }^{[1,2]}$. However, dose distribution depends on several factors such as treatment planning method and dose calculation algorithms employed in treatment planning systems (TPS). Furthermore, dose calculation algorithms face challenge in predicting accurate dose when inhomogenous media are encountered along the photon beam path. For example, in the case of esophageal cancer treatment, there is an involvement of lung (low-density medium), which causes the electronic disequilibrium effect near the air/tissue interfaces. Thus, when an esophageal target is to be irradiated using external beam radiation therapy, dose calculation algorithms must apply tissue heterogeneity corrections that will account accurately for the electron transport near tissue heterogeneity interface.

Recently, a new dose calculation algorithm called Acuros XB (AXB) was implemented in the Eclipse TPS (Varian Medical Systems, Palo Alto, CA). A number of studies have evaluated the dosimetric impact of AXB for different clinical sites ${ }^{[3-8]}$ by comparing the dosimetric results of AXB with that of Anisotropic Analytical Algorithm (AAA). Most of the previous dosimetric studies on $\mathrm{AXB}{ }^{[3-8]}$ were based on physical dose-volume statistics, which can characterize only the certain aspects of the 3D dose distribution. Furthermore, other researchers ${ }^{[9-11]}$ have investigated the use of RapidArc technique for esophageal cancer, but the evaluation of Varian's most recent dose calculation algorithm AXB using radiobiological parameters for esophageal cancer remains to be done.

The main purpose of this study was to perform the radiobiological response model comparison between AXB and AAA for esophageal cancer treatment plans. The radiobiological evaluation was done using tumor control probability (TCP), which provides a quantitative biophysical measure of tumor dose ${ }^{[12]}$. The TCP was estimated using the numerically calculated equivalent uniform dose (EUD) ${ }^{[13]}$ obtained from the dose distributions of esophageal cancer treatment plans created by RapidArc technique.

\section{Methods}

\subsection{Dose Calculation algorithms}

Since the validation of AXB has already been by several researchers ${ }^{[14-17]}$, no comparisons between the AXB calculations and measurements were done in this study. The AAA is based on a pencil-beam convolution/superposition technique, and the primary photons, scattered photons and electrons scattered from the beam limiting devices are modeled separately in $\mathrm{AAA}^{[18]}$. The tissue heterogeneity in the AAA is handled by scaling of primary photons and photon scatter kernel scaling in lateral directions according to local electron density ${ }^{[18-20]}$. A more detailed description of the AAA is presented elsewhere ${ }^{[18-20]}$. The AXB solves numerically Linear Boltzmann Transport Equation which describes the macroscopic behavior of radiation particles as they travel through and interact with matter ${ }^{[14]}$. The AXB is considered to be similar to classic Monte Carlo methods for accurate modeling of dose deposition in heterogeneous media ${ }^{[14]}$. A more detailed description of the AXB is presented elsewhere ${ }^{[14,15]}$.

\subsection{Treatment planning}

Ten esophageal cancer cases treated at Arizona Center for Cancer Care were selected for the current study. This study was approved by the research committee of the institution. The computed tomography (CT) scans of all cases were acquired with $512 \times 512$ pixels at $0.25 \mathrm{~cm}$ slice spacing on a flat tabletop of a GE LightSpeed CT Scanner. The planning target volume (PTV) was created using a $5 \mathrm{~mm}$ wide isotropic expansion of the clinical target volume (CTV). The organs at risk (OARs), such as the lungs, heart, liver, and spinal cord, were delineated based on the axial CT images. All cases were planned using RapidArc technique with two arcs (Figure 1) and 6X mode of the $600 \mathrm{MU} / \mathrm{min}$ dose rate in the Eclipse TPS (version 10.0.28). The treatment plans were inversely optimized using progressive resolution optimizer (PRO), version 
10.0.28, such that at least $95 \%$ of the PTV received the prescription dose of 50.4 Gy in 28 fractions. The optimized plans were calculated using AAA with tissue heterogeneity correction, and each calculated plan was then normalized such that $100 \%$ of the prescribed dose covered $95 \%$ of the PTV. The final plan generated after plan normalization was referred as the AAA plan. The AXB plan was generated by recalculating the final AAA plan using AXB with tissue heterogeneity correction for identical monitor units and beam parameters as in the corresponding AAA plan. The calculation grid was set to $2.5 \mathrm{~mm}$ for all cases.

Figure 1. A transversal view of RapidArc plan setup in the Eclipse treatment planning system for esophageal cancer (case \#8). The planning target volume (PTV) is shown in the above figure.

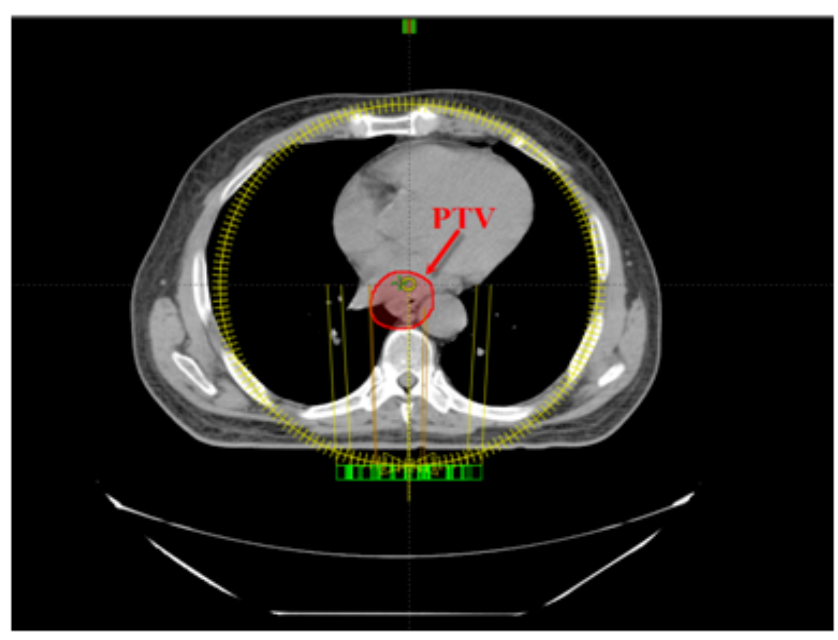

\subsection{Equivalent uniform dose}

The EUD is defined as the dose which causes the same biological effect if distributed homogeneously throughout the entire volume of the structure of interest as the inhomogeneous dose distribution ${ }^{[13,21]}$.

According to Niemierko's phenomenological model, the equation for EUD ${ }^{[13,21,22]}$ is given as

$$
E U D=\left(\sum_{i=1}\left(v_{i} E Q D_{i}^{a}\right)\right)^{\frac{1}{a}}
$$

In equation (1), $a$ is a unit less model parameter that is specific to the normal structure or tumor of interest, and $v_{i}$ is unit less and represents the $i^{\text {th }}$ partial volume receiving dose $D_{i}$ in Gy ${ }^{[13,21,22]}$. Since the relative volume of the whole structure of interest corresponds to 1 , the sum of all partial volumes $v_{i}$ will equal $1^{[13,21,22]}$. Furthermore, in equation (1), the $\mathrm{EQD}^{[22]}$ is the biologically equivalent physical dose of $2 \mathrm{~Gy}$ and defined as:

$$
E Q D=D \times \frac{\left(\frac{\alpha}{\beta}+\frac{D}{n_{f}}\right)}{\left(\frac{\alpha}{\beta}+2\right)}
$$

Where, $\mathrm{n}_{\mathrm{f}}$ and $\mathrm{d}_{f}=\mathrm{D} / \mathrm{n}_{\mathrm{f}}$ are the number of fractions and dose per fraction size of the treatment course, respectively. The $\alpha / \beta$ is the tissue-specific linear-quadratic (LQ) parameter of the organ being exposed ${ }^{[13,21]}$. The EUD calculations were performed using the MATLAB and Simulink Student Version-R2012a (The MathWorks, Inc., Natick, Massachusetts). 
First, the cumulative dose-volume histograms (DVHs) of AAA and AXB plans were exported from the Eclipse TPS using dose bin size of 40 cGy. Second, we utilized the MatLab program ${ }^{[21]}$ to calculate the EUD with the Niemierko method ${ }^{[13,21]}$. For the esophageal cancer, the EUD was calculated using alpha-beta ratio of $10^{[22]}$. The alpha-beta ratio for lung, liver, heart, and spinal cord were 3, 1.5, 2, and 2, respectively ${ }^{[22,23]}$. For complete list of EUD parameters, please refer to Table 1.

\subsection{Tumor control probability}

The concept of TCP is important in radiobiological modeling, which can be used in raking different treatment plans that lead to same biological endpoint. The TCP model was developed on the basis that after irradiation of target, some cells received one or more hits and die, whereas some cells do not get any hit and survive ${ }^{[12]}$. This random killing of clonogenic cells by radiations is well described by Poisson statistics ${ }^{[22]}$. Using LQ expression for cell survival, TCP was calculated as:

$$
\mathrm{TCP}=\mathrm{e}^{-\mathrm{N}\left(\mathrm{SF}_{2}\right)} \frac{\frac{\mathrm{D}}{\text { Dref }}}{\left(\frac{\frac{\alpha}{\beta}+\frac{\mathrm{D}}{\mathrm{n}}}{\frac{\alpha}{\beta}+\text { Dref }}\right)}
$$

Where, $\mathrm{N}=$ the number of clonogen tumor cells, calculated by multiplying clonogenic cell density (CCD) by tumor volume, alpha/beta $=10$ was used, where alpha and beta are radiosensitivity parameters related to cell killing from single hit or multiple hit events respectively. $\mathrm{D}$ equals EUD, $\mathrm{n}$ equals the number of treatment fractions, and $\mathrm{SF}_{2}$ is the surviving fraction after irradiation at a reference dose $\left(\mathrm{D}_{\text {ref }}\right)$ of $2 \mathrm{~Gy}$. The probability of cells surviving at $2 \mathrm{~Gy}$ was assumed to be 0.5 , and TCP calculations were based on CCD of $10 \mathrm{M} / \mathrm{cc}$.

Table 1. Parameters used to calculate EUD using Niemierko's method

\begin{tabular}{lllllll}
\hline Tissue & Volume Type & $\mathbf{1 0 0 \%} \mathbf{d p f}$ & \#f & a & Dpf (Gy) & $\boldsymbol{\alpha} / \boldsymbol{\beta}(\mathbf{G y})$ \\
\hline Esophagus (PTV) & Tumor & 1.8 & 28 & -13 & 2 & 10 \\
Lung & Normal & 1.8 & 28 & 1 & 2 & 3 \\
Liver & Normal & 1.8 & 28 & 3 & 2 & 1.5 \\
Heart & Normal & 1.8 & 28 & 3 & 2 & 2 \\
Spinal Cord & Normal & 1.8 & 28 & 13 & 2 & 2 \\
\hline
\end{tabular}

$100 \% \mathrm{dpf}=100 \%$ dose per fraction; $\# \mathrm{f}=$ number of fractions; $\alpha / \beta=$ alpha-beta ratio; and dpf= parameters' source data's dose per fraction; EUD = Equivalent Uniform Dose, PTV=planning target volume. (The parameters are obtained from published papers of Oinam et al. ${ }^{[22]}$, Kehwar ${ }^{[23]}$, and Okunieff et al. ${ }^{[25]}$ )

\subsection{Analysis}

For the purpose of comparison, the percent difference, D (\%), in the EUD or TCP values between the AXB and AAA plans was calculated using equation (4).

$$
\mathrm{D}(\mathrm{x})=\left(\frac{\mathrm{AXB}-\mathrm{AAA}}{\mathrm{AAA}}\right) \times 100
$$

where $\mathrm{x}$ is the EUD or TCP value in the AXB and AAA plans for the same case.

\section{Results}

Tables 2-6 shows the EUD values of ten cases of esophageal cancer for the PTV and OARs in the AAA and AXB plans. In this section, the EUD results from AAA and AXB calculations are expressed as average \pm standard deviation inside the parentheses. 
Table 2. EUD for the PTV in the RapidArc esophageal cancer treatment plans (AXB \&AAA)

\begin{tabular}{llll}
\hline \multirow{2}{*}{ Case \# } & EUD (Gy) & D (\%) \\
\cline { 2 - 3 } & AXB & AAA & -1.5 \\
2 & 50.3 & 51.1 & -1.5 \\
3 & 50.4 & 51.2 & -1.3 \\
4 & 50.4 & 51.1 & -1.8 \\
5 & 50.2 & 51.1 & -0.9 \\
6 & 50.8 & 51.3 & -1.7 \\
7 & 50.3 & 51.1 & -0.9 \\
8 & 52.2 & 52.6 & -0.7 \\
9 & 52.1 & 52.5 & -1.6 \\
10 & 51.3 & 52.2 & -1.3 \\
Avg. & 50.5 & 51.2 & -1.3 \\
SD. & 50.9 & 51.5 & 0.4 \\
\hline
\end{tabular}

EUD = Equivalent Uniform Dose, AAA = Anisotropic Analytical Algorithm, AXB = Acuros XB Algorithm; Avg. = Average, $\mathrm{SD}=$ Standard Deviation; D $(\%)=[(\mathrm{AXB}-\mathrm{AAA}) / \mathrm{AAA}] \times 100$

Table 3. EUD for the lung in the RapidArc esophageal cancer treatment plans (AXB \&AAA)

\begin{tabular}{llll}
\hline \multirow{2}{*}{ Case \# } & EUD (Gy) & AAA & D (\%) \\
\cline { 2 - 4 } & AXB & 4.8 & -4.6 \\
\hline 1 & 4.6 & 6.9 & -4.4 \\
2 & 6.6 & 6.6 & -4.1 \\
3 & 6.4 & 7.3 & -3.2 \\
4 & 7.1 & 4.8 & -3.6 \\
5 & 4.6 & 3.8 & -4.9 \\
6 & 3.6 & 5.7 & -2.5 \\
7 & 5.5 & 4.6 & -2.5 \\
8 & 4.5 & 4.2 & -2.4 \\
9 & 4.1 & 7.4 & -2.6 \\
10 & 7.2 & 5.6 & -3.5 \\
Avg. & 5.4 & 1.3 & 1.0 \\
SD. & 1.3 & & \\
\hline
\end{tabular}

EUD $=$ Equivalent Uniform Dose, $\mathrm{AAA}=$ Anisotropic Analytical Algorithm, AXB $=$ Acuros XB Algorithm; Avg. $=$ Average, $\mathrm{SD}=\mathrm{Standard}$ Deviation; $\mathrm{D}(\%)=[(\mathrm{AXB}-\mathrm{AAA}) / \mathrm{AAA}] \times 100$

Table 4. EUD for the liver in the RapidArc esophageal cancer treatment plans (AXB \&AAA)

\begin{tabular}{llll}
\hline \multirow{2}{*}{ Case \# } & EUD (Gy) & AAA & D (\%) \\
\cline { 2 - 4 } & AXB & 11.3 & -2.3 \\
1 & 11.1 & 12.2 & -3.1 \\
2 & 11.8 & 8.3 & -2.3 \\
3 & 8.1 & 7.1 & -2.6 \\
5 & 6.9 & 16.0 & -1.4 \\
6 & 15.8 & 14.2 & -3.1 \\
7 & 13.8 & 15.4 & -1.8 \\
8 & 15.1 & 9.7 & -1.9 \\
9 & 9.5 & 11.1 & -2.5 \\
10 & 10.8 & 5.7 & -1.9 \\
Avg. & 5.6 & 11.1 & -2.3 \\
SD. & 10.8 & 3.5 & 0.5 \\
\hline
\end{tabular}

EUD = Equivalent Uniform Dose, AAA = Anisotropic Analytical Algorithm, AXB = Acuros XB Algorithm; Avg. = Average, SD = Standard Deviation; D $(\%)=[(\mathrm{AXB}-\mathrm{AAA}) / \mathrm{AAA}] \times 100$ 
For the PTV, the EUD values in the AXB plans $(50.9 \pm 0.8 \mathrm{~Gy})$ were always lower than in the AAA plans $(51.5 \pm 0.6 \mathrm{~Gy})$ with an average difference of $1.3 \%$. Similar to the results for the PTV, the AXB calculations produced smaller magnitude of EUD for the OARs compared to the AAA calculations. On average, in the AXB plans, the lung EUD was lower by $3.5 \%$ ( $5.4 \pm 1.3$ Gy vs. $5.6 \pm 1.3 \mathrm{~Gy})$, the liver EUD was lower by $2.3 \%$ ( $10.8 \pm 3.4 \mathrm{~Gy}$ vs. $11.1 \pm 3.5 \mathrm{~Gy})$, the heart EUD was lower by $2.8 \%(20.7 \pm 6.7$ Gy vs. $21.3 \pm 6.8 \mathrm{~Gy})$, and the spinal cord EUD was lower by $1.8 \%(18.7 \pm 6.8$ Gy vs. $19.1 \pm 6.9 \mathrm{~Gy})$.

Table 5. EUD for the heart in the RapidArc esophageal cancer treatment plans (AXB \&AAA)

\begin{tabular}{llll}
\hline \multirow{2}{*}{ Case \# } & EUD (Gy) & D (\%) \\
\cline { 2 - 4 } & AXB & AAA & -2.2 \\
\hline 1 & 24.0 & 24.5 & -2.8 \\
2 & 20.7 & 21.3 & -2.7 \\
3 & 23.6 & 24.2 & -2.9 \\
4 & 28.4 & 29.2 & -1.5 \\
5 & 27.0 & 27.4 & -2.6 \\
6 & 14.7 & 15.1 & -3.6 \\
7 & 12.1 & 12.6 & -3.3 \\
8 & 10.7 & 11.0 & -3.0 \\
9 & 17.2 & 17.7 & -3.1 \\
10 & 28.7 & 29.6 & -2.8 \\
Avg. & 20.7 & 21.3 & 0.6 \\
SD. & 6.7 & 6.8 & \\
\hline
\end{tabular}

EUD $=$ Equivalent Uniform Dose, AAA = Anisotropic Analytical Algorithm, AXB = Acuros XB Algorithm; Avg. $=$ Average, $\mathrm{SD}=\mathrm{Standard}$ Deviation; $\mathrm{D}(\%)=[(\mathrm{AXB}-\mathrm{AAA}) / \mathrm{AAA}] \times 100$

The analysis of TCP also showed different results for the AXB (74.2 $\pm 5.9 \%)$ and AAA (79.8 $\pm 4.1 \%$ ) plans (Table 7). For each case, the TCP in the AXB plan was always lower than in the AAA plan. On average, the TCP of AXB plans was lower by $7.1 \%$ (range, $2.5 \%-10.6 \%$ ) compared to that of AAA plans.

Table 6. EUD for the spinal cord in the RapidArc esophageal cancer treatment plans (AXB \&AAA)

\begin{tabular}{llll}
\hline \multirow{2}{*}{ Case \# } & EUD (Gy) & & D (\%) \\
\cline { 2 - 4 } & AXB & AAA & -1.9 \\
\hline 1 & 22.0 & 22.4 & -1.1 \\
2 & 19.6 & 19.8 & -1.8 \\
3 & 26.7 & 27.2 & -2.2 \\
4 & 27.2 & 27.8 & -1.8 \\
5 & 17.4 & 17.7 & -1.9 \\
6 & 16.9 & 17.2 & -2.9 \\
7 & 11.6 & 11.9 & -2.5 \\
8 & 7.3 & 7.5 & -1.4 \\
9 & 13.0 & 13.2 & -0.8 \\
10 & 25.9 & 26.1 & -1.8 \\
Avg. & 18.7 & 19.1 & 0.6 \\
SD. & 6.8 & 6.9 & \\
\hline
\end{tabular}

$\mathrm{EUD}=$ Equivalent Uniform Dose, $\mathrm{AAA}=$ Anisotropic Analytical Algorithm, AXB = Acuros XB Algorithm; Avg. = Average, $\mathrm{SD}=\mathrm{Standard}$ Deviation; $\mathrm{D}(\%)=[(\mathrm{AXB}-\mathrm{AAA}) / \mathrm{AAA}] \times 100$ 
Table 7. TCP for the PTV in the RapidArc esophageal cancer treatment plans (AXB \&AAA)

\begin{tabular}{llll}
\hline \multirow{2}{*}{ Case \# } & \multicolumn{2}{l}{ TCP (\%) } & D $(\%)$ \\
\cline { 2 - 4 } & AXB & 76.8 & -9.3 \\
1 & 69.7 & 77.6 & -9.0 \\
3 & 70.7 & 76.8 & -8.0 \\
4 & 70.7 & 76.8 & -10.6 \\
5 & 68.7 & 78.4 & -5.2 \\
6 & 74.3 & 76.8 & -9.3 \\
7 & 69.7 & 86.5 & -2.5 \\
8 & 84.3 & 85.9 & -2.6 \\
9 & 83.7 & 84.3 & -7.0 \\
10 & 78.4 & 77.6 & -7.7 \\
Avg. & 71.6 & 79.8 & -7.1 \\
SD. & 74.2 & 4.1 & 2.8 \\
\hline
\end{tabular}

$\mathrm{TCP}=$ Tumor Control Probability, AAA = Anisotropic Analytical Algorithm, AXB = Acuros XB Algorithm; Avg. = Average, SD = Standard Deviation; D $(\%)=[(\mathrm{AXB}-\mathrm{AAA}) / \mathrm{AAA}] \times 100$

\section{Discussion}

The accurate modeling of primary beam attenuation and lateral scatter is important in photon dose calculation algorithms in order to avoid the dose overestimation or underestimation. However, due to the difference in beam modeling approach within dose calculation algorithms, the discrepancies in their dose predictions exist, especially when photon beam traverses the inhomogenous media before it reaches the tumor. A number of studies ${ }^{[14-17]}$ have reported the superiority of the AXB over the AAA for dose predictions when heterogeneous media are involved.

In the current study, the highest average difference (3.5\%) in EUD was seen for the lung, which involves the low density medium that causes the electronic disequilibrium. In previous studies ${ }^{[15,17]}$, the AAA was shown to predict the dose higher than the AXB in the low density air gap region. If the AXB is considered to be more accurate than the AAA, the current study showed that the AAA may be overestimating the magnitude of EUD for the lung. Moreover, the preliminary results from the current study showed the EUD values were always lower in the AXB plans compared with the AAA plans. In contrast, the results of AXB were not always lower in magnitude compared with those of AAA in the dosimetric study of esophageal cancer treatment plans ${ }^{[7]}$. Specifically, the AXB predicted lower mean and minimum PTV dose but a higher maximum PTV dose ${ }^{[7]}$. Similarly, the maximum doses to the OARs in the AXB plans were lower for the heart, liver, and spinal cord but higher for the lung compared to the AAA plans ${ }^{[7]}$. The results from current study demonstrated that comparison between the AXB and AAA may result different outcomes depending on the treatment plan evaluation method. Furthermore, all esophageal treatment plans in this study were generated using same technique (i.e., RapidArc planning) that we currently use at Arizona Center for Cancer Care. The use of different planning techniques may provide different dosimetric and radiobiological results for AAA and AXB. For example, Vivekanandan et al. ${ }^{[9]}$ performed the dosimetric comparison between 3D-conformal radiation therapies, 4-field intensity modulated radiation therapy, and single-arc and double-arc RapidArc plans of esophageal cancer. It would be interesting to investigate if the discrepancies between the results of AAA and AXB calculations would be same for different planning techniques.

In the current study, the radiobiological modeling analysis for tumor control showed that the AAA may be overestimating the TCP values compared to the AXB. In the current study, we assumed that probability of cells surviving at 2 Gy to be 0.5 ; however, population of clonogenic cells may differ in their radioresistance ${ }^{[26]}$. Thus, the probability distribution of cells surviving at 2 Gy may vary. Furthermore, TCP was evaluated for single CCD. But, the CCD may vary from patient to patient and may also differ within the tumor volume of the same patient. The TCP calculation on AXB and AAA plans using various $\mathrm{CCD}$ and SF2 will be an interesting topic for future studies. 


\section{Conclusion}

In comparison to the AAA calculations, the AXB calculations produced lower magnitudes of EUD and TCP for the target volume as well as lower EUD for the critical structures (lung, liver, heart, and spinal cord) in the esophageal cancer treatment plans.

\section{Conflicting interests}

The authors declare that they have no conflicting interests.

\section{References}

[1] Otto K. Volumetric modulated arc therapy: IMRT in a single gantry arc. Med Phys. 2008; 35: 310-7. PMid:18293586 http://dx.doi.org/10.1118/1.2818738

[2] Ling CC, Zhang P, Archambault Y, Bocanek J, Tang G, Losasso T. Commissioning and quality assurance of RapidArc radiotherapy delivery system. Int J Radiat Oncol Biol Phys. 2008; 72: 575-81. PMid:18793960 http://dx.doi.org/10.1016/j.ijrobp.2008.05.060

[3] Han T, Mourtada F, Kisling K, Mikell J, Followill D, Howell R. Experimental validation of deterministic Acuros XB algorithm for IMRT and VMAT dose calculations with the Radiological Physics Center's head and neck phantom. Med Phys. 2012; 39: 2193-202.

[4] Kan MW, Leung LH, Yu PK. Verification and dosimetric impact of Acuros XB algorithm on intensity modulated stereotactic radiotherapy for locally persistent nasopharyngeal carcinoma. Med Phys. 2012; 39: 4705-14. PMid:22894395 http://dx.doi.org/10.1118/1.4736819

[5] Fogliata A, Nicolini G, Clivio A, Vanetti E, Cozzi L. On the dosimetric impact of inhomogeneity management in the Acuros XB algorithm for breast treatment. Radiat Oncol. 2011; 6: 103. PMid:21871079 http://dx.doi.org/10.1186/1748-717X-6-103

[6] Fogliata A, Nicolini G, Clivio A, Vanetti E, Cozzi L. Critical appraisal of Acuros XB and Anisotropic Analytic Algorithm dose calculation in advanced non-small-cell lung cancer treatments. Int J Radiat Oncol Biol Phys. 2012; 83: 1587-95. PMid:22300575 http://dx.doi.org/10.1016/j.ijrobp.2011.10.078

[7] Rana S, Rogers K, Pokharel S, Lee T, Reed D, Biggs C. Acuros XB Algorithm vs. Anisotropic Analytical Algorithm: A Dosimetric Study Using Heterogeneous Phantom and Computed Tomography (CT) Data Sets of Esophageal Cancer Patients. J Cancer Ther. 2013; 4: 138-144. http://dx.doi.org/10.4236/jct.2013.41019

[8] Rana S, Rogers K, Lee T, Reed D, Biggs C. Verification and Dosimetric Impact of Acuros XB Algorithm for Stereotactic Body Radiation Therapy (SBRT) and RapidArc Planning for Non-Small-Cell Lung Cancer (NSCLC) Patients. Int Jour of Med Phys Clin Eng and Rad Onc. 2013; 2: 6-14. http://dx.doi.org/10.4236/ijmpcero.2013.21002

[9] Vivekanandan N, Sriram P, Kumar SA, Bhuvaneswari N, Saranya K. Volumetric modulated arc radiotherapy for esophageal cancer. Med Dosim. 2012; 37: 108-13. PMid:21940159 http://dx.doi.org/10.1016/j.meddos.2011.01.008

[10] Sriram P, Syamkumar SA, Kumar JS, Prabakar S, Dhanabalan R, Vivekanandan N. Adaptive volumetric modulated arc treatment planning for esophageal cancers using cone beam computed tomography. Phys Med. 2012; 28: 327-32. PMid:22079402 http://dx.doi.org/10.1016/j.ejmp.2011.10.006

[11] Martin S, Chen JZ, Rashid Dar A, Yartsev S. Dosimetric comparison of helical tomotherapy, RapidArc, and a novel IMRT \& Arc technique for esophageal carcinoma. Radiother Oncol. 2011; 101: 431-7. PMid:21962823 http://dx.doi.org/10.1016/j.radonc.2011.08.030

[12] Niemierko A, Goitein M. Implementation of a model for estimating tumor control probability for an inhomogeneously irradiated tumor. Radiother Oncol. 1993; 29: 140-7. http://dx.doi.org/10.1016/0167-8140(93)90239-5

[13] Niemierko A. Reporting and analyzing dose distributions: a concept of equivalent uniform dose. Med Phys. 1997; $24: 103-10$. PMid:9029544 http://dx.doi.org/10.1118/1.598063

[14] Vassiliev ON, Wareing TA, McGhee J, Failla G, Salehpour MR, Mourtada F. Validation of a new grid based Blotzmann equation solver for dose calculation in radiotherapy with photon beams. Phys Med Biol. 2010; 55: 581-98. PMid:20057008 http://dx.doi.org/10.1088/0031-9155/55/3/002

[15] Bush K, Gagne IM, Zavgorodni S, Ansbacher W, Beckham W. Dosimetric validation of Acuros XB with Monte Carlo methods for photon dose calculations. Med Phys. 2011; 38: 2208-21. PMid:21626955 http://dx.doi.org/10.1118/1.3567146

[16] Fogliata A, Nicolini G, Clivio A, Vanetti E, Cozzi L. Dosimetric evaluation of Acuros XB Advanced Dose Calculation algorithm in heterogeneous media. Radiat Oncol. 2011; 6: 82. PMid:21771317 http://dx.doi.org/10.1186/1748-717X-6-82 
[17] Rana S, Rogers K. Dosimetric evaluation of Acuros XB dose calculation algorithm with measurements in predicting doses beyond different air gap thickness for smaller and larger field sizes. J Med Phys. 2013; 38: 9-14. PMid:23532180 http://dx.doi.org/10.4103/0971-6203.106600

[18] Tillikainen L, Helminen H, Torsti T, Siljamäki S, Alakuijala J, Pyyry J, Ulmer W. A 3D pencil-beam-based superposition algorithm for photon dose calculation in heterogeneous media. Phys Med Biol. 2008; 53: 3821-39. PMid:18583728 http://dx.doi.org/10.1088/0031-9155/53/14/008

[19] Ulmer W, Harder D. Applications of a triple gaussian pencil beam model for photon beam treatment planning. Med Phys. 1996; 6: 68-74.

[20] Van Esch A, Tillikainen L, Pyykkonen J, Tenhunen M, Helminen H, Siljamäki S, et al. Testing of the analytical anisotropic algorithm for photon dose calculation. Med Phys. 2006; 33: 4130-48. PMid:17153392 http://dx.doi.org/10.1118/1.2358333

[21] Gay HA, Niemierko A. A free program for calculating EUD-based NTCP and TCP in external beam radiotherapy. Phys Med. 2007; 23: 115-25. PMid:17825595 http://dx.doi.org/10.1016/j.ejmp.2007.07.001

[22] Oinam AS, Singh L, Shukla A, Ghoshal S, Kapoor R, Sharma SC. Dose volume histogram analysis and comparison of different radiobiological models using in-house developed software. J Med Phys. 2011; 36: 220-9. PMid:22228931 http://dx.doi.org/10.4103/0971-6203.89971

[23] Kehwar TS. Analytical approach to estimate normal tissue complication probability using best fit of normal tissue tolerance doses into the NTCP equation of the linear quadratic model. J Cancer Res Ther. 2005; 1: 168-79. PMid:17998649 http://dx.doi.org/10.4103/0973-1482.19597

[24] Tucker SL, Thames HD, Taylor JM. How well is the probability of tumor cure after fractionated irradiation described by Poisson statistics? Radiat Res. 1990; 124: 273-82. PMid:2263727 http://dx.doi.org/10.2307/3577839

[25] Okunieff P, Morgan D, Niemierko A, Suit HD. Radiation dose-response of human tumors. Int J Radiat Oncol Biol Phys. 1995; 32: 1227-37. http://dx.doi.org/10.1016/0360-3016(94)00475-Z

[26] Baumann M, Krause M, Hill R. Exploring the role of cancer stem cells in radioresistance. Nat Rev Cancer. 2008 ; 8: 545-54. PMid:18511937 http://dx.doi.org/10.1038/nrc2419 